\title{
Importância da tomografia de córnea para o diagnóstico de ectasia
}

\author{
Importance of anterior segment tomography \\ for the diagnosis of corneal ectasia
}

Bruno de Freitas Valbon ${ }^{1,3}$, Marcella Salomão ${ }^{2,3}$, Isaac Ramos ${ }^{3}$, Ana Laura Canedo ${ }^{3}$, Leonardo Nogueira3, Renato Ambrósio Jr ${ }^{3}$

\begin{abstract}
ResUMO
Objetivo: Utilização da metodologia para interpretação da tomografia de córnea e segmento anterior para a deteç̧ão e diagnóstico de ectasia corneana. Métodos: Descrição da interpretação clínica dos índices da tomografia de córnea e segmento anterior (Pentacam High Resolution - Oculus, Wetzlar,Germany); relato de 3 casos, demonstrando a importância da propedêutica no exame complementar, através do Pentacam HR®. Conclusão: Nossa experiência compatibiliza-se com os relatos de literatura na demonstração do incremento de sensibilidade e especificidade para a detecção precoce de susceptibilidade e diagnóstico de ectasia corneana, utilizando a tomografia de córnea e segmento anterior.
\end{abstract}

Descritores: Ectasia/diagnóstico; Tomografia; Córnea; Ceratocone

\section{ABSTRACT}

Purpose: The methodology currently used for interpretation of the cornea and anterior segment tomography for the diagnosis of corneal ectasia and its susceptibility.Methods: Description of the methodology and clinical interpretation of corneal and anterior segment tomography indexes; report of three cases demonstrating the importance of this new diagnostic tool (Pentacam HR $®$ ) in ophthalmological practice.Conclusion: The use of corneal and anterior segment tomography seems to be an effective method to increase the sensitivity and specificity for the diagnosis and early detection of corneal ectasia.

Keywords: Ectasia/diagnosis; Tomography; Cornea; Keratoconus

'Pós Graduando - Nível Doutorado da Universidade de São Paulo, USP, Brasil;

2Programa de Pós-graduação (Doutorado) Escola Paulista de Medicina, Universidade Federal de São Paulo (UNIFESP) - São Paulo (SP), Brasil;

${ }^{3}$ Grupo de Estudos de tomografia e biomecânica de córnea do Rio de Janeiro - Rio de Janeiro (RJ), Brasil.

Os autores declaram inexistir conflitos de interesse

Recebido para publicação em 4/8/2011 - Aceito para publicação em 18/5/2012 


\section{INTRODUÇÃO}

$\mathbf{0}$ advento da cirurgia refrativa determinou uma necessidade de evolução de métodos avançados de screening e diagnóstico de ectasia. Um dos aspectos préoperatórios mais importantes para candidatos à Lasik é saber qual é o risco de susceptibilidade para ectasia.

A ectasia corneana progressiva é uma complicação rara, porém grave, sendo uma das principais causas de processos contra médicos nos EUA ${ }^{(1,2)}$. Fatores de risco para ectasia pósLasik são bastante conhecidos na literatura, porém ainda envoltos em controvérsias ${ }^{(3)}$. O diagnóstico pré-operatório de ceratocone e degeneração marginal pelúcida são obviamente importantes fatores de risco, especialmente nas apresentações subclínicas, conjuntamente com o leito estromal residual, que deve ser suficiente para a manutenção da integridade da arquitetura corneana.

Os cirurgiões refrativos enfrentam rotineiramente o desafio de identificar adequadamente os casos de maior risco para ectasia. Tradicionalmente, a topografia da córnea baseada na superfície anterior através dos discos de plácido e a espessura central corneana (ECC) têm sido ainda determinantes para análise de susceptibilidade de ectasia ${ }^{(4-6)}$ pela grande maioria dos cirurgiões, pois são considerados como métodos de screening importantes em pacientes candidatos à cirurgia refrativa, como demonstra o clássico critério do ectasia risk scoring system (ERSS) desenvolvido por Randleman et al. ${ }^{(7)}$. Porém, este critério apresenta limitações, como mostrou o estudo de Chan et al. ${ }^{(8)}$ que apresentou uma série de 36 casos de ectasia pós-Lasik, onde 9 olhos $(25 \%)$ apresentavam baixo risco e 7 olhos (19\%) moderado risco, de acordo com os critérios de Randleman, para o desenvolvimento de ectasia. Assim, há uma necessidade inquestionável para novas ferramentas de diagnóstico que irão complementar o importante mapa de curvatura da superfície anterior da córnea (topografia) e avaliação da espessura central corneana para o desenvolvimento de uma abordagem mais sensível e específica para a identificação de susceptibilidade e diagnóstico de ectasia.

A tomografia de córnea $a^{(9)}$ por meio dos mapas de elevação anterior e posterior, o perfil de progressão paquimétrica e os índices tomográficos, como ART (Ambrosio Relational Thinnest) e parâmetro D (deviation) podem ajudar-nos a buscar e diferenciar córneas normais e doentes, ou susceptíveis à doença.

Ambrosio et al. ${ }^{(10)}$ em artigo aceito para publicação no Journal Refractive Surgery em 2011, descreveram o conceito do Ambrosio Relational Thinnest (ART) que avalia o ponto mais fino sobre os índices de progressão paquimétrica. Este estudo demonstrou que a curva ROC para o ART Average e Maximum são 0,987 e 0,983, respectivamente e o limite numérico sugerido destes parâmetros, para diferenciar olhos normais e com ceratocone é de 424 e 339, respectivamente. Em outro estudo (Ambrosio e cols. apresentam dados ainda não publicados de 2010) foi demonstrado que para diferenciar córneas saudáveis e com ceratocone, o número limítrofe sugerido do valor D (Parâmetro D) é 1,6.

Nesse artigo, demonstramos casos interessantes em que a análise da tomografia, dos mapas e de índices tomográficos possibilitam identificar sinais de susceptibilidade numa córnea com topografia de aspecto normal, córnea normal com suspeita de ceratocone e córnea doente com topografia de aspecto normal.

\section{Métodos}

Utilização da propedêutica da tomografia de córnea e segmento anterior na avaliação de candidatos à cirurgia refrativa e os seus métodos de screening utilizados para detecção precoce e diagnóstico de ectasia.

Caso 1 - Apresentamos um caso de ectasia pós-Lasik em um olho com risco baixo para ectasia, segundo os critérios de Randleman e análise tomográfica do olho contralateral determinando a susceptibilidade para ectasia, caso viesse a realizar a técnica Lasik.

Caso 2 - Neste segundo caso há uma avaliação de uma suspeita de ectasia pela topografia corneana, no qual, os índices topográficos detectaram $90,1 \%$ de chance de ser ceratocone. E ao analisarmos este mesmo olho com a tomografia de córnea e os índices apresentados, aumentamos a especificidade, confirmado o "verdadeiro negativo", ou seja, excluindo o diagnóstico de ceratocone.

Caso 3 - Neste terceiro caso há uma demonstração da importância da tomografia de córnea na detecção de sinais e índices de ectasia numa córnea com topografia normal, apresentando índices de inteligência artificial para normal de 45,93\%; e others* (Tabela 1) com 54,07\%. Ao estudarmos os dados tomográficos neste caso, aumentamos a sensibilidade ao afirmarmos os aspectos característicos de ectasia corneana.

Tabela 1

\section{Identificação de siglas}

\begin{tabular}{l}
\hline Tabela de Identificação das Siglas dos Parametros estudados neste artigo \\
\hline BAD display-The Belin/Ambrosio Enhanced Ectasia Display \\
\hline CTSP-Corneal Thickness Spatial Profile \\
PTI-Percentual Thickness Increase \\
\hline ART-Ambrosio's Relational Thinnest. \\
\hline TP-Thinnest Point \\
\hline PPI-Pachymetric Progression Index \\
\hline BFS-Best Fit Sphere \\
\hline D - Deviation \\
\hline $\begin{array}{l}\text { *OTHERS-Corneal Topography has the characteristics associated w ith } \\
\text { unclassified variations }\end{array}$ \\
\hline
\end{tabular}

\section{Caso 1- Topografia e paquimetria normal evoluindo com ectasia pós-Lasik}

\section{Dados clínicos e exames complementares}

Mulher, 31 anos, veio para segunda opinião, devido à insatisfação com a visão após Lasik em OE há quatro anos.

Refração pré-operatório: OD -6.00 esf -1.00 cil 180 (20/15) e OE -5.75 esf -1.25 cil a 10 (20/15). A espessura central corneana era de 528 micras em ambos os olhos. Foi realizada a cirurgia refrativa pela técnica de Lasik em OE com microcerátomo, com espessura de flap de $160 \mu \mathrm{m}$ e com ablação de $81 \mu \mathrm{m}$. Não foram coletados dados intraoperatórios da espessura do flap e estroma residual. Paciente evoluiu bem com 20/25 de visão sem correção, porém insatisfeita com a qualidade da visão se queixava de halos. Foi realizada topografia documentada após duas semanas com perfil de ablação de astigmatismo miópico centrado. Insatisfeita com a qualidade de visão do $\mathrm{OE}$, a paciente decidiu não realizar a cirurgia do OD, preferindo a utilização de lente de contato.

Após 4 anos, notou piora da visão do OE e nos foi referida para avaliação e tratamento. Na figura 1, observamos a face anterior da córnea com a topografia corneana do OD através da imagem de Scheimpflug com ceratometria simulada 44.70@166.9/45.20, não havendo índices topográficos de ceratocone evidentes. Ao 


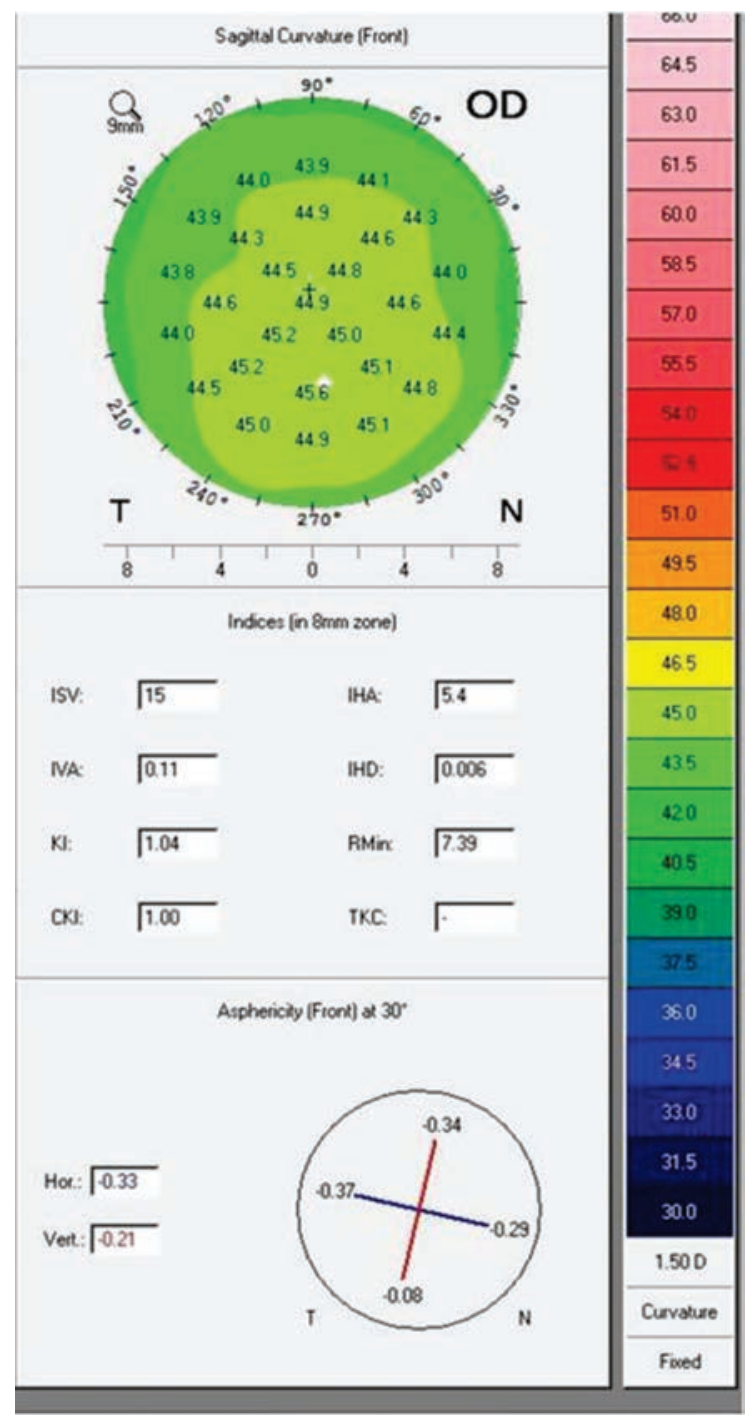

Figura 1: Topografia corneana do OD não observamos alterações e/ ou índices topográficos para ceratocone e/ou ectasia

realizarmos a topografia corneana em dois olhos com discos de plácido (Magellan Navigator Corneal Topographer®; Nidek), diagnosticamos OE como ectasia pós-Lasik (Figura 2). Segundo, os critérios de Randleman ERRS ${ }^{(11)}$, o risco de ectasia foi calculado como baixo ( 2 pontos) antes da cirurgia de Lasik em OE. Caso considerássemos, Lasik para OD e assim utilizarmos os mesmos critérios de Randleman (ERRS) para o OD, encontraríamos score como baixo risco para ectasia (1 ponto). Porém, ao avaliarmos este OD com a tomografia de córnea e segmento anterior com imagem de Scheimpflug, através do software Belin/Ambrosio Enhanced Display (BAD) (Figura 3), observamos os mapas de elevação anterior e posterior e este último se encontra levemente alterado. Ao visualizarmos o mapa diferencial ou de subtração, observamos padrões dentro da normalidade, "caminhando" para a zona verde (normal) que apresenta limite de $12 \mu \mathrm{m}$.

Ao analisarmos, os gráficos de progressão paquimétrica, principalmente o Percentual Thickness Increase (PTI), descrito por Ambrosio et al. ${ }^{(12)}$, percebemos um escape nos $4.5 \mathrm{~mm}$ do intervalo de confiança de 95\%, confirmando uma distribuição

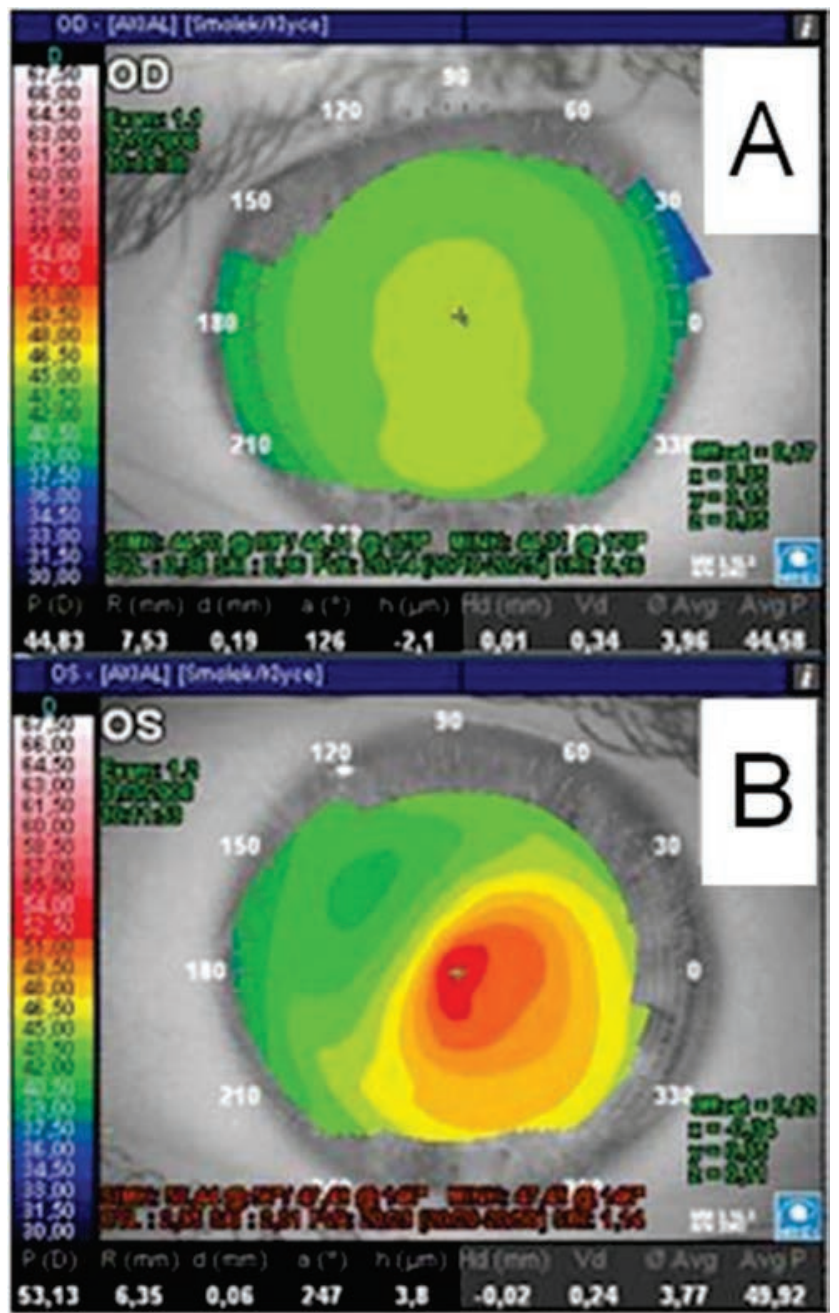

Figura 2: Mapas de curvatura axial, utilizando a escala absoluta de Smolek-Klyce; nota-se a córnea com asfericidade normal e baixa toricidade em OD (A); em OE (B) observa-se ectasia temporal inferior, os índices de inteligência artificial do Nidek corneal navigator identifica normalidade em OD (NRM $=62.2 \%$; OTH $=32.8 \%$; KC, KCS and $\mathrm{PMD}=0 \%)$ e ceratocone $\mathrm{em} \mathrm{OE}(\mathrm{KC}=99 \%, \mathrm{KSI}=13.1 \%)$

paquimétrica anormal. Ao estudar os índices deviation (parameter $D)$ do $B A D$, Ambrósio e cols. (dados não publicados, 2010), demonstraram que o melhor cut off para diferenciar olhos com susceptibilidade à ectasia e normais é de 1,6. Este paciente apresenta no BAD (Figura 3) um $D$ (parameters deviation) de 1,99, classificado como suspeito de ectasia.

Com esta análise dos índices tomográficos do OD, podemos perceber que esta paciente apresenta, apesar de um ART Max de 424, alguns aspectos importantes para susceptibilidade à ectasia como PTI e parâmetro $D$ alterados e se pensarmos que esta mesma paciente evoluiu com ectasia pós-Lasik em OE, poderíamos evitar esta complicação, caso avaliássemos previamente este olho com a tomografia de córnea analisando a curva de progressão paquimétrica (PTI) e o valor D (parameter D-deviation).

\section{Caso 2 - Topografia suspeita com bow tie assimétrico em ambos os olhos}

\section{Dados clínicos e exames complementares}

Mulher, 39 anos compareceu para avaliação com suspeita 
de ceratocone. Apresentava uma acuidade visual de 20/20 sem correção em AO. Biomicroscopia sem sinais de ectasia. Apresentava, espessura central corneana de 590 e 588 micras em $\mathrm{OD}$ e $\mathrm{OE}$, respectivamente. Ao analisarmos a topografia corneana de ambos os olhos (Figura 4), notamos um bow tie assimétrico, e ao visualizarmos os índices de inteligência artificial, percebemos que o OE apresentava uma suspeita de ceratocone de 90,1\%.A ceratometria em OE era de K1 45,21@ 176 e K2 46.29 @ 86. Sugerimos realizar a tomografia de córnea para avaliarmos o aspecto tridimensional da córnea (faces anterior e posterior), e assim aumentarmos a nossa especificidade com este teste diagnóstico, estudando os índices tomográficos de ambos os olhos. O Display Belin/Ambrosio (BAD) permite esta avaliação mais criteriosa, principalmente da face posterior, curva de progressão paquimétrica e índices como ART Max e o Parameter $D$. Este caso se torna muito interessante, pois temos um olho (OE) com aspecto topográfico de ceratocone e os índices de inteligência artificial do topógrafo apontam grande chance de ectasia, porém ao estudarmos os aspectos tomográficos pelo BAD do OE (Figura 5), não observamos nenhum sinal de ectasia e nem de susceptibilidade à ectasia. Os mapas apresentaram diferenciais tanto da elevação anterior quanto da posterior estando dentro da normalidade. Ao visualizarmos a curva de progressão paquimétrica (PTI), notamos que está dentro do padrão de distribuição normal, não havendo escape nem para cima, nem para baixo num intervalo de confiança de $95 \%$. Ao calcularmos o ART Max (ponto mais fino sobre o índice de progressão máximo), encontramos o valor de 554,71, bem acima do cut off (339), tendo uma margem bem elevada de segurança. O valor D é 0.90 , se afastando da população de ceratocone, segundo o artigo de Ambrosio et al. ${ }^{(10)}$. Estes dados tomográficos permitiu-nos aumentarmos a segurança e especificidade, ao excluirmos assim, a doença nesta paciente.

\section{Caso 3 - Topografia normal, com características tomográficas de doença ectásica}

\section{Dados clínicos e exames complementares}

Homem, 24 anos apresentando diagnóstico de ceratocone assimétrico e acuidade visual com correção em OD 20/20 (-3.00 esf -200 cil a 20$)$ e OE 20/20 (-0.75 cil a 9). A espessura central corneana em OD é de 505 micras e de 535 micras em OE.

Na Figura 6, observamos a topografia corneana em OD e OE e notamos aspecto de ectasia inferior em OD, caracterizando ceratocone. Ao observarmos em OE, percebemos uma topografia normal, com índices de inteligência artificial de 45,6\% NRM (Normal Cornea) e 54,4\% "Others". O Display Belin-Ambrosio (BAD) deste olho esquerdo apresenta características tomográficas semelhantes à doença ectásica, apesar de topo normal sem sinais de ectasia.

Na figura 7, o BAD do olho esquerdo, nota-se um valor D de 1.59, acima do cut off da normalidade; ao observarmos o PTI, visualizamos um escape da curva desde os $4,5 \mathrm{~mm}$ e percebemos que a mesma percorre no limite inferior do desvio padrão no intervalo de $95 \%$ de confiança. O cálculo do ART Max é de 359, bem próximo ao cut off para ceratocone (339). Estes dados demonstram na análise por tomografia, córneas com aspectos topográficos normais, e assim podemos aumentar a sensibilidade com esta avaliação tomográfica e detectarmos sinais característicos de córnea doente.
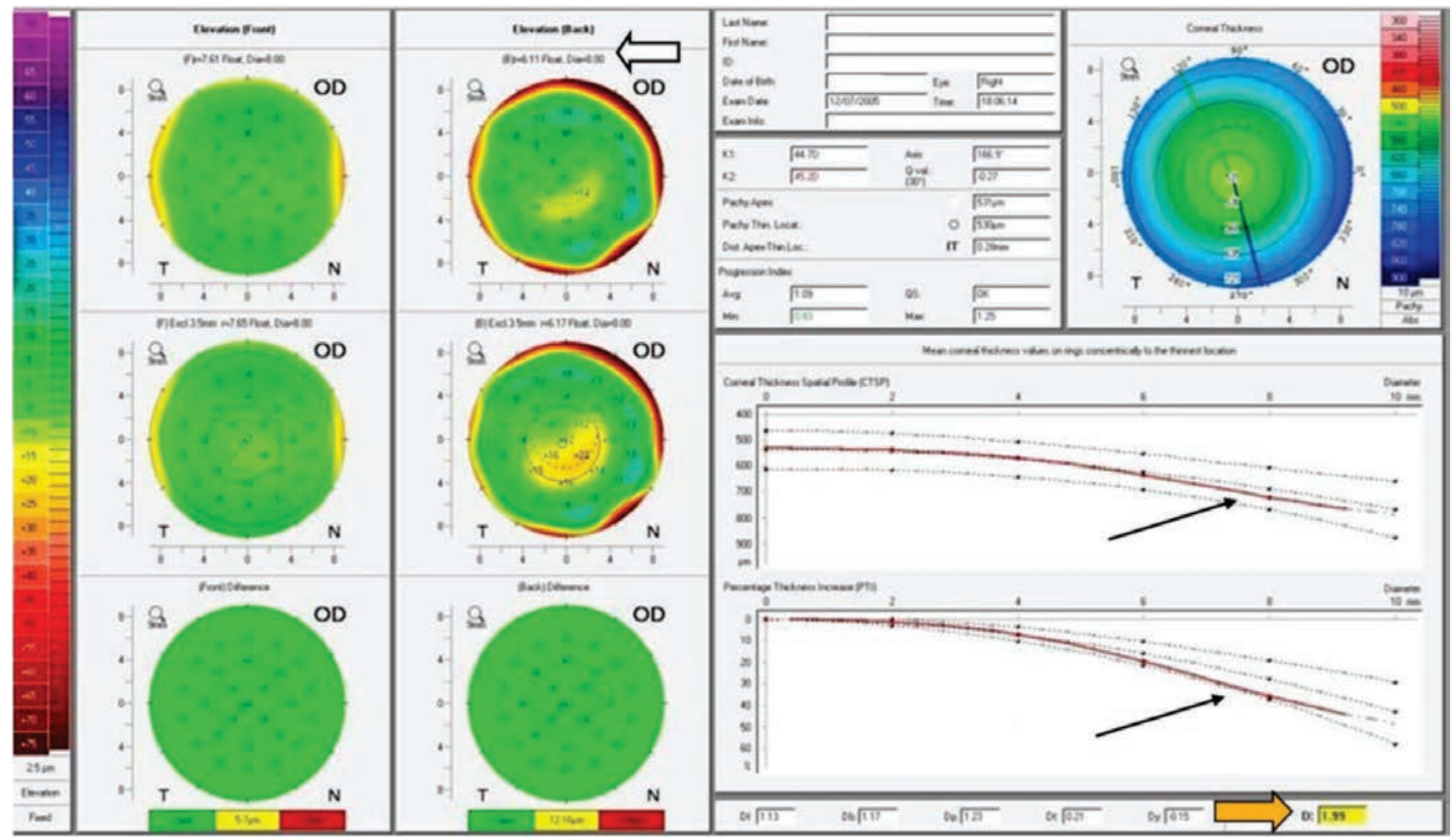

Figura 3: Belin / Ambrosio Display Enhanced Ectasia; índices tomográficos com os mapas de elevação anterior e posterior, curva de progressão paquimétrica, índices deviation (D), índices de progressão paquimétrica; observação: seta laranja significa o valor D; seta preta identifica o escape da curva de progressão paquimétrica (CTSP e PTI); seta branca mostra os mapas de elevação posterior com o melhor BFS de 8.0 mm 


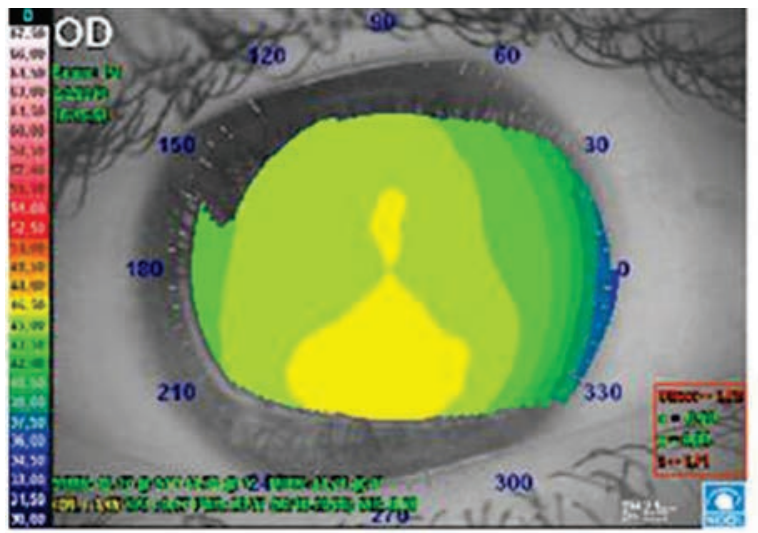

o Avg (mm) S.4.
Nymm) 0.96

VImmi:0.20

Ang $P(D): 45,41$

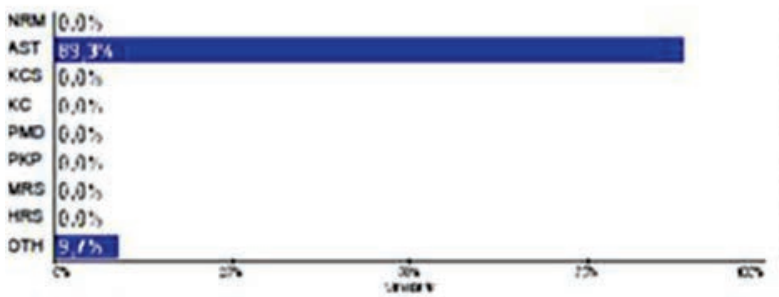

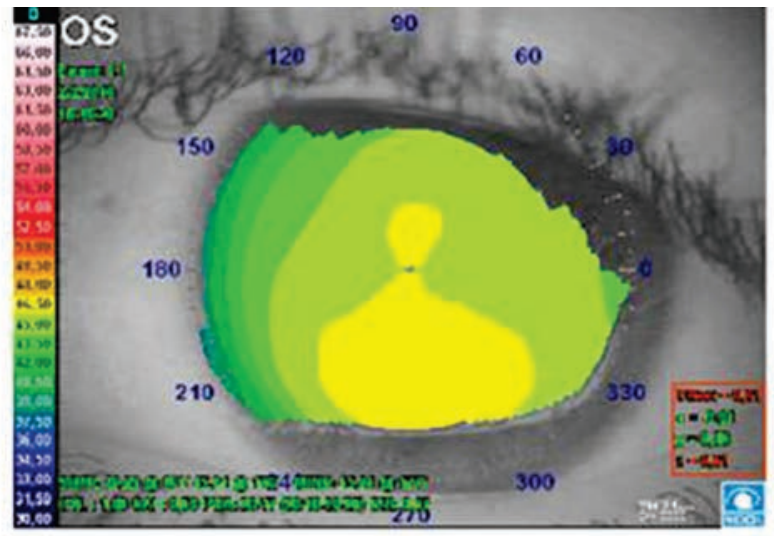

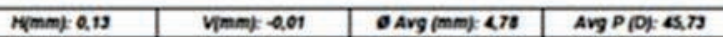

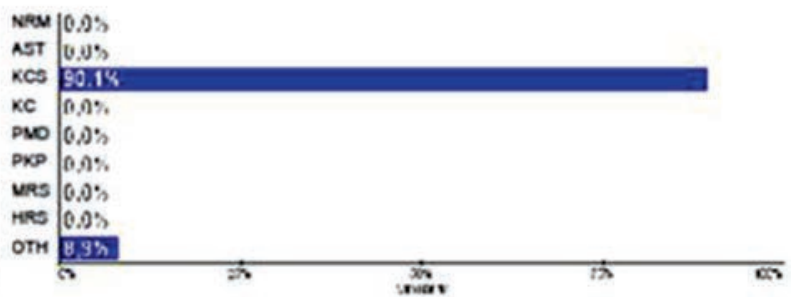

Figura 4: Topografia corneana com aspecto de um bow tie assimétrico em ambos os olhos; os índices de inteligência artificial do Nidek corneal topographer magellan navigator identifica em OD, 89,3 \% de astigmatismo e em OE 90,1\% de KCS (suspeita de ceratocone)

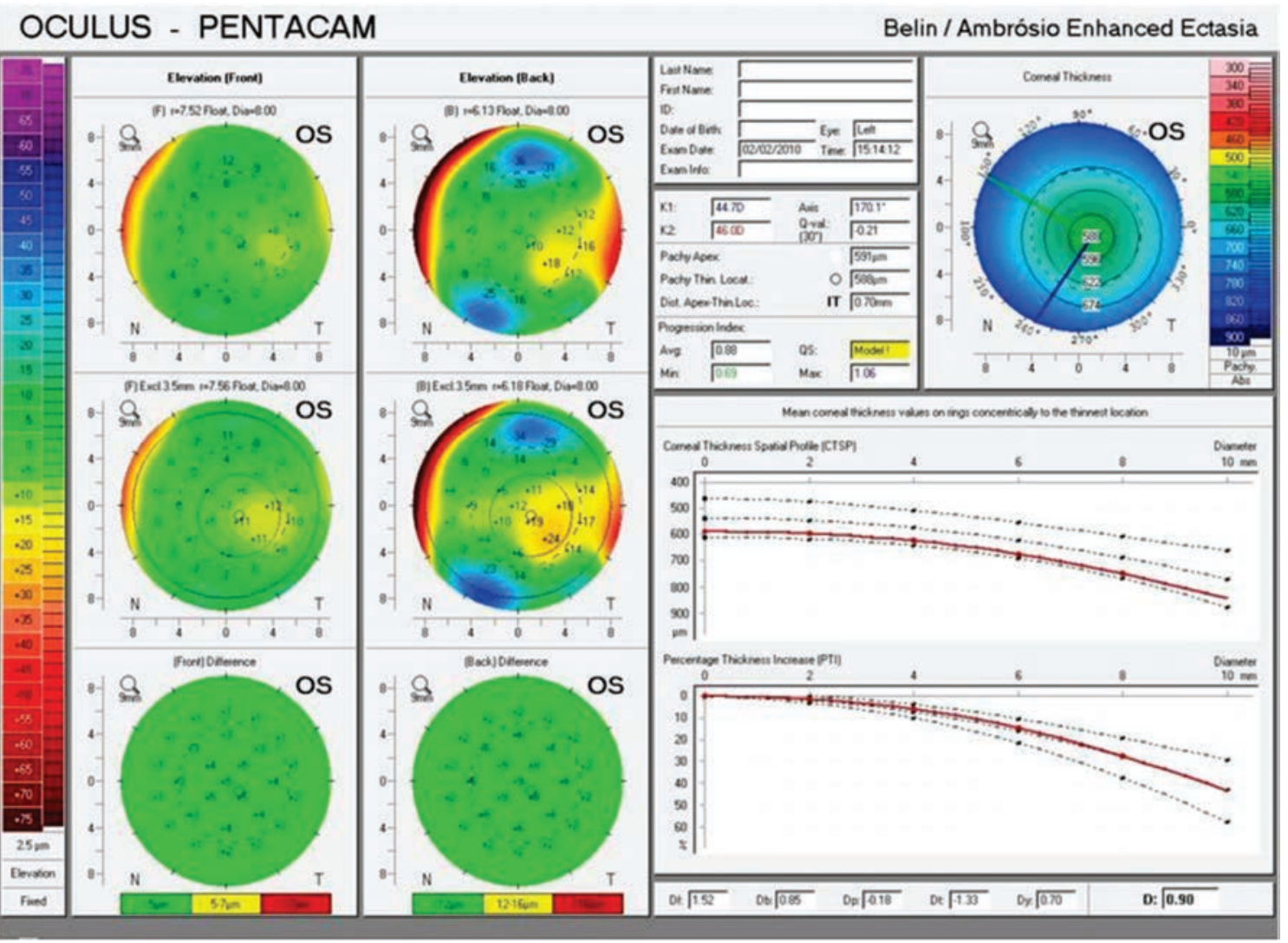

Figura 5: Display Belin- Ambrosio (BAD); mapas diferenciais de elevação anterior e posterior normais; a curva de progresão paquimétrica percentage of thickness increase (PTI) apresenta normalidade, não havendo nenhum escape dentro do desvio padrão médio (intervalo de confiança 95\%); o valor D (parameter- deviation) é 0.90, bem abaixo do cut off $(1,6)$ entre normais e ceratocones. O ART Máx (TP/PPI Max) é de 554,71,com isso, podemos excluir doença ectásica 


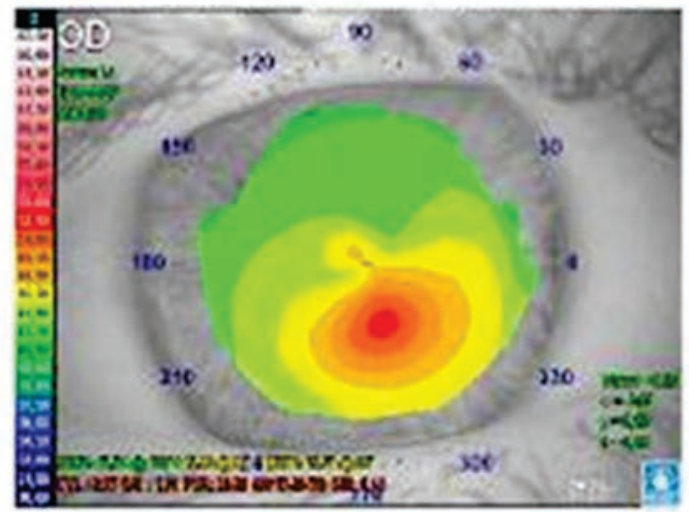

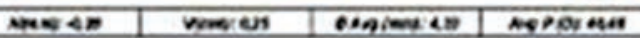

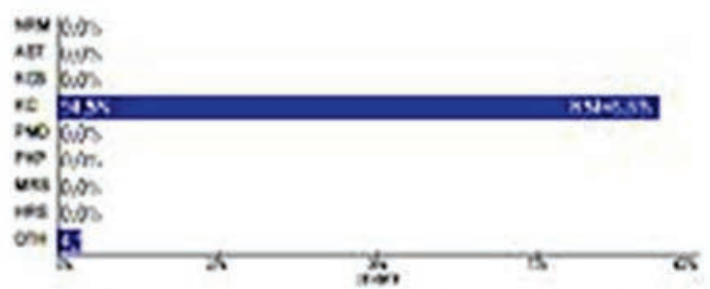

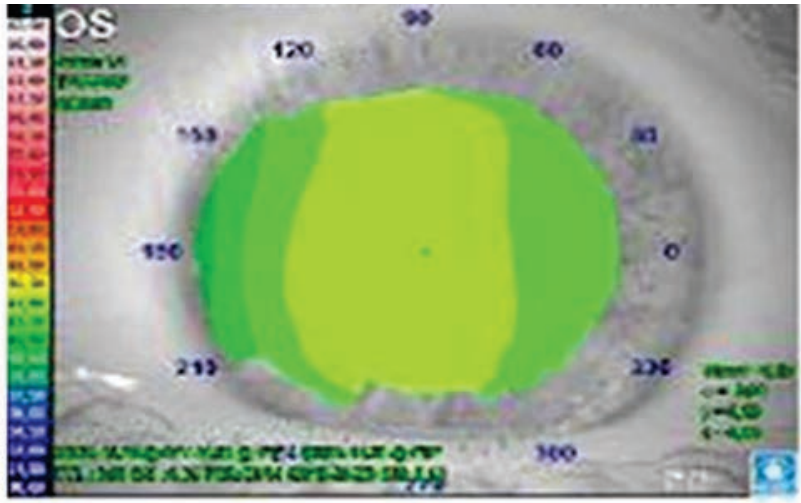

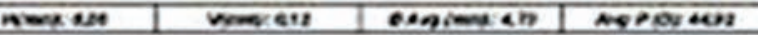

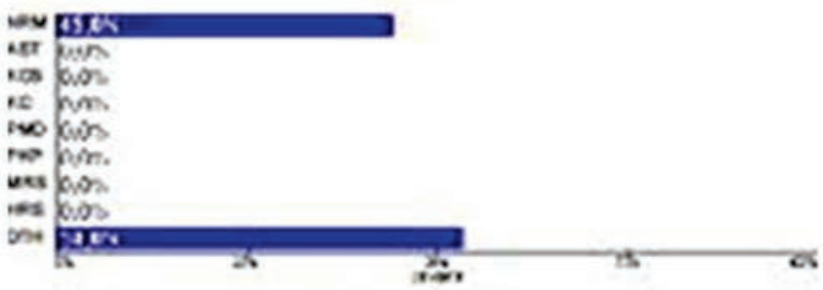

Figura 6: Topografia com aspecto de ectasia inferior; logo abaixo, notamos o olho contralateral deste paciente com topografia normal, e índices de inteligência artificial dividido entre normal e outros

OCULUS - PENTACAM

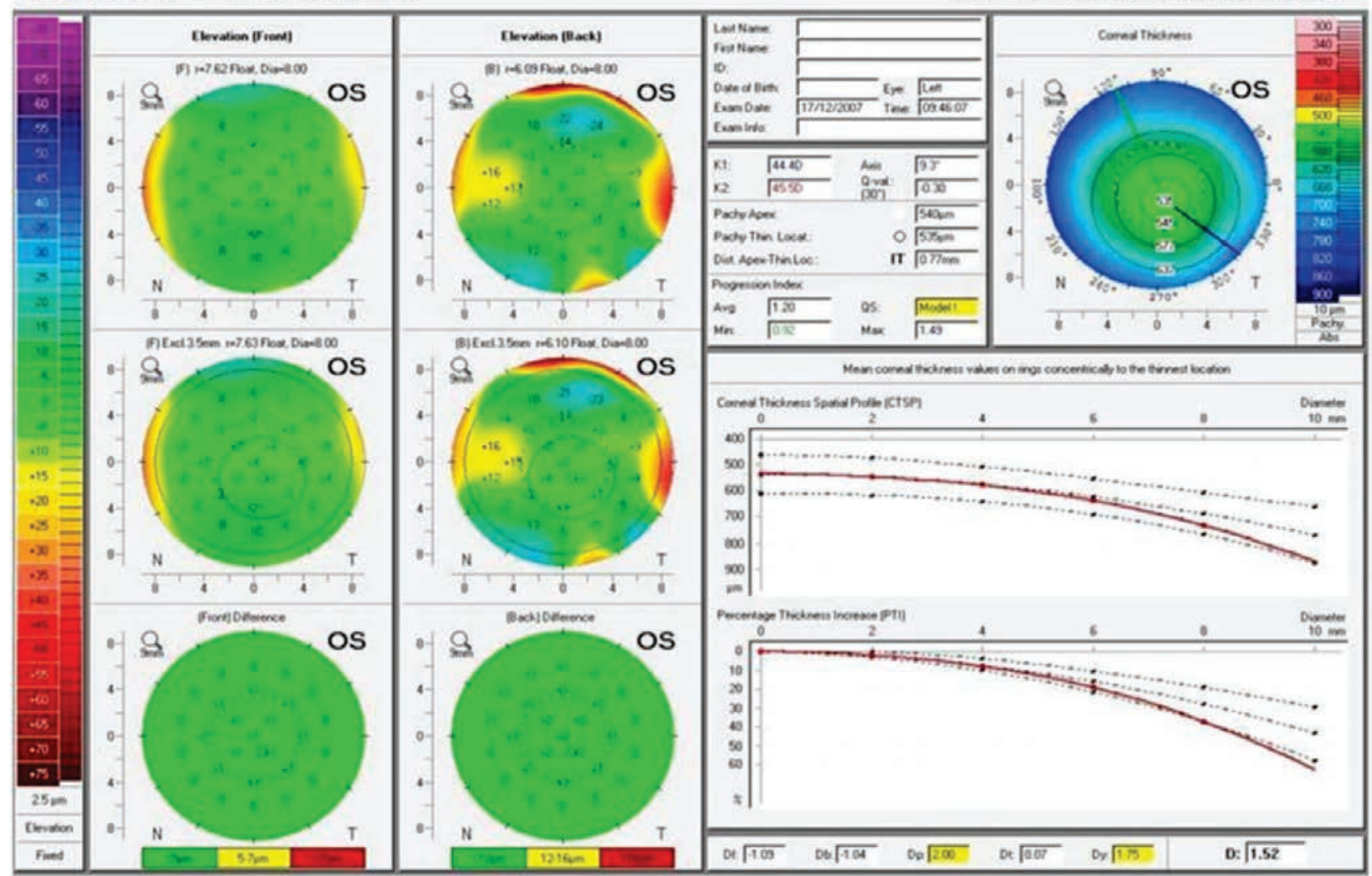

Figura 7: Display Belin Ambrosio (BAD) - Analisamos o olho contralateral de um cone assimétrico com aspecto topográfico normal, conforme na figura 6; Notamos, curva de progressão paquimétrica (PTI), ART Máx de 359 e Valor D de 1,52, índices alterados característicos de córnea doente 


\section{DiscusSÃo}

Desde o advento da topografia corneana ${ }^{(13)}$, que estuda detalhadamente a face anterior da córnea, novas tecnologias incrementam a propedêutica para caracterização da córnea em nível tomográfico ${ }^{(14)}$, biomecânico ${ }^{(15)}$, bem como das aberrações de alta ordem do sistema ótico ocular ${ }^{(16)}$. Variáveis geradas por estes exames mais avançados permitem, além de aumentar nossa capacidade de diagnóstico e prognóstico de ceratocone e outras ectasias, melhorarmos o planejamento cirúrgico e o seguimento dos resultados obtidos.

Embora a ectasia pós-lasik ${ }^{(1,2)}$ seja rara (e mesmo havendo protocolos de screening como, o ERSS, desenvolvido por Randleman ${ }^{(11)}$, o primeiro caso que demonstramos reforça a necessidade de desenvolvimento de melhores testes de triagem.

A atual evolução das técnicas de diagnóstico como a tomografia em $3 \mathrm{D}^{(14)}$, o estudo da biomecânica e análise de aberrações de alta ordem, podem ajudar o cirurgião a melhorar a interpretação e precisão de seleção de candidatos à Lasik.

Belin/Ambrosio Enhanced Display (BAD) ${ }^{(9)}$ é a primeira ferramenta completa para diagnóstico de ectasia corneana e sua susceptibilidade, baseados em mapas de elevação anterior e posterior. O objetivo deste software é ajudar na identificação dos pacientes que podem ser susceptíveis e/ou têm a doença em estágio inicial ou subclínica e até mesmo excluir casos suspeitos. No caso 1, podemos identificar algumas características tomográficas de córnea susceptível à ectasia, podendo assim evitar uma possível complicação. Tentamos demonstrar nos casos 2 e 3, que este display foi fundamental para caracterizarmos córneas saudáveis com topografia suspeita e ao mesmo tempo córnea susceptível com topografia normal, respectivamente. Sendo assim, utilizando informações dos mapas de elevação anterior e posterior, do perfil paquimétrico e os índices tomográficos, como o ART e parameter $\mathrm{D}$, podemos melhorar a sensibilidade e especificidade para diferenciarmos córneas normais, doentes e susceptíveis à ectasia.

De Sanctis et al. ${ }^{(17)}$ em publicação recente demonstraram que ao analisar a elevação posterior da córnea através da tomografia (Pentacam) houve um aumento da sensibilidade e especificidade para diferenciação entre córneas normais e ceratocones. Uçakhan et al. ${ }^{(18)}$ em um trabalho semelhante mostraram que ao analisar os índices tomográficos é possível discriminar pacientes com ceratocone e normais.

Logo, estudos futuros e em larga escala serão necessários e fundamentais para que tenhamos testes mais sensíveis e específicos e assim, critérios seletivos mais fidedignos. O estudo da biomecânica da córnea pode nos ajudar a entender melhor o surgimento da ectasia e de córneas susceptíveis, sendo importante analisarmos não somente a arquitetura e estrutura corneana, mas também a sua viscoelasticidade.

\section{ReferênCIAS}

1. Seiler T, Quurke AW. Iatrogenic keratectasia after LASIK in a case of forme fruste keratoconus. J Cataract Refract Surg. 1998;24(7):1007-9. Comment in J Cataract Refract Surg. 2000;26(5):629-30.

2. Binder PS, Lindstrom RL, Stulting RD, Donnenfeld E, Wu H, McDonnell P, Rabinowitz Y. Keratoconus and corneal ectasia after LASIK. J Refract Surg. 2005;21(6):749-52.

3. Tabbara KF, Kotb AA. Risk factors for corneal ectasia after LASIK. Ophthalmology. 2006;113(9):1618-22.

4. Ambrósio R Jr, Klyce SD, Wilson SE. Corneal topographic and pachymetric screening of keratorefractive patients. J Refract Surg. 2003;19(1):24-9.
5. Oshika T, Klyce SD. Corneal topography in LASIK. Semin Ophthalmol. 1998;13(2):64-70. Review.

6. Ambrósio R Jr, Wilson SE. Complications of laser in situ keratomileusis: etiology prevention, and treatment. J Refract Surg. 2001;17(3):350-79. Comment in J Refract Surg. 2001;17(4):475.

7. Randleman JB, Woodward M, Lynn MJ, Stulting RD. Risk assessment for ectasia after corneal refractive surgery. Ophthalmology. 2008;115(1):37-50.

8. Chan CC, Hodge C, Sutton G. External analysis of the Randleman Ectasia Risk Factor Score System: a review of 36 cases of post LASIK ectasia. Clin Experiment Ophthalmol. 2010;38(4):335-40.

9. Belin MW, Khachikian SS, Ambrosio R Jr, Salomão M. Keratoconus/Ectasia Detection with the Oculus Pentacam: Belin/Ambrosio Enhaced Ectasia Display. Highligths Ophtahlmol. 2007;35(6):5-8.

10. Ambrosio R Jr, Canedo AL, Guerra FP, Louzada R, Roy AS, Luz A, et al. Novel pachymetric parameters based on corneal tomography for diagnosing keratoconus. J Refract Surg. 2011;27(10): 753-8.

11. Randleman JB, Trattler WB, Stulting RD. Validation of the Ectasia Risk Score System for preoperative laser in situ keratomileusis screening. Am J Ophthalmol. 2008;145(5):813-8.

12. Ambrósio R Jr, Alonso RS, Luz A, Coca Velarde LG. Cornealthickness spatial proûle and corneal-volume distribution: tomographic indices to detect keratoconus. J Cataract Refract Surg. 2006;32(11):1851-9.

13. Klyce SD. Computer-assisted corneal topography. High-resolution graphic presentation and analysis of keratoscopy. Invest Ophthalmol Vis Sci. 1984;25(12):1426-35.

14. Ambrósio R Jr, Belin MW. Imaging of the cornea: topography vs tomography. J Refract Surg. 2010;26(11):847-9.

15. Luce DA. Determining in vivo biomechanical properties of the cornea with an ocular response analyzer. J Cataract Refract Surg. 2005;31(1):156-62.

16. Wilson SE, Ambrosio R. Computerized corneal topography and its importance to wavefront technology. Cornea. 2001;20(5):441-54.

17. de Sanctis U, Loiacano C, Richiardi L, Turco D, Mutani B, Grignolo FM. Sensitivity and specificity of posterior corneal elevation measured by Pentacam in discriminating Keratoconus/subclinical Keratoconus. Ophthalmology. 2008;115(9):1534-9.

18. Uçakhan öö, Centikor V, Özkan M, Kanpolat A. Evaluation of Scheimplufg imaging parameters in subclinical Keratoconus, keratoconus, and normal eyes. J Cataract

Autor correspondente:

Bruno de Freitas Valbon

Av. Beira Mar, n 2767 - apto 102 - Bairro Bento Ferreira -

CEP 29052-121 - Vitória (ES), Brasil

Tel: (21) 8103-7117

E-mail: valbonbruno@ig.com.br 